DOI: https://doi.org/10.46502/issn.2710-995X/2020.4.06

\title{
Causas de muerte y hallazgos necrópsicos en fallecidos por cirrosis hepática durante el decenio de 1991-2000 en Santiago de Cuba
}

\section{Causes of death and necropsy findings in deceased by Liver Cirrhosis during the decade 1991-2000 in Santiago de Cuba}

\author{
Escrito por: \\ María Isabel Castillo Duany ${ }^{14}$ \\ https://orcid.org/0000-0002-2802-7868
}

\section{Resumen}

Se realizó un estudio descriptivo, transversal y retrospectivo con el objetivo de describir las principales causas de muerte y hallazgos necrópsicos en fallecidos por Cirrosis Hepática en el Hospital Provincial Clínico Quirúrgico Docente "Saturnino Lora", en Santiago de Cuba, durante el decenio de 1991-2000. Los resultados demostraron pérdidas de años de vida potencialmente perdidos y el incremento progresivo de la frecuencia de necropsia, de la mortalidad a partir de los 55 años con predominio del sexo masculino y trabajadores, así como el aumento de la mortalidad en los primeros siete días de estadía hospitalaria. La insuficiencia hepática aguda y la bronconeumonía bacteriana resultaron ser las principales causas directas de muerte y como resultado relevante en los hallazgos necrópsicos se destacaron la ascitis, esplenomegalia y ateroesclerosis de la aorta y sus ramas. En la clasificación etiológica de los fallecidos la Cirrosis Portal y la post-necrótica constituyeron las más representativas.

Palabras Claves: Cirrosis Hepática, hallazgos necrópsicos, insuficiencia hepática aguda.

\section{Abstract}

A descriptive, transversal and retrospective study was carried out with the aim of describing the main causes of death and necropsy findings in patients who died of liver cirrhosis at the "Saturnino Lora" Provincial Clinical and Surgical Teaching Hospital, in Santiago de Cuba, during the decade 1991-2000. The results showed losses of potentially lost years of life and the progressive increase in the frequency of necropsy, of the mortality from 55 years of age with predominance of male sex and workers, as well as the increase of mortality in the first seven days of hospital stay. Acute liver failure and bacterial bronchopneumonia were the main direct causes of death, and ascites, splenomegaly and atherosclerosis of the aorta and its branches were relevant in the necropsy findings. In the etiological classification of the deceased, portal cirrhosis and post-necrotic cirrhosis were the most representative.

\footnotetext{
${ }^{14}$ Especialista de Primer Grado de Medicina Interna. Policlínico Comunitario Alberto Fernández Montes de Oca, San Luis, Santiago de Cuba.
} 
Key words: Liver Cirrhosis, necropsy findings, acute liver failure.

\section{Introducción}

La Cirrosis Hepática tiene una distribución amplia a nivel mundial y afecta todas las razas. Los datos epidemiológicos obtenidos de la Organización Mundial de la Salud a partir de 55 países excepto la República Popular de China y la antigua República Socialista Soviética, indicaron que generalmente morían cada año en el mundo más de 310000 personas por esta enfermedad (Martini \& Bodec., 1970). La gran masa de información estadística sobre la misma como problema sanitario se basa en el acuerdo de codificación de la International Clasification of Disease (LCD).

Un punto débil e importante en este tipo de información estadística es la realidad cuestionable del diagnóstico de Cirrosis como causa de muerte. De ahí, que los diferentes tipos de Cirrosis estaban deficientemente representados en las diferentes categorías de la LCD, ya que esto se basaba esencialmente en datos morfológicos post-morten solamente. La tasa de supervivencia de cinco años de los pacientes enfermos por dicha causa era comparable con los que estaban afectados por diversos procesos neoplásicos. Así, por ejemplo, se había comunicado una supervivencia a los tres años del $16 \%$ y a los cinco años solo el $8 \%$ en un grupo de 308 pacientes una vez hecho el diagnóstico (Creutzteldt \& Beek, 1956).

La etiología de la Cirrosis varía en función de factores tanto geográficos como sociales. En el mundo occidental, la frecuencia aproximada de las categorías etiológicas era la siguiente: Hepatopatíaalcohólica (60-70\%); Hepatitis Viral (10\%); Enfermedades biliares (5-10\%); Hemocromatosis primaria (5\%), Déficit de la alfa 1 antitripsina (rara), Enfermedad de Wilson (rara) y Cirrosis (10-15\%). Una vez establecida la Cirrosis el diagnóstico etiológico suele ser difícil de precisar solo a partir de los hallazgos morfológicos. (Van \& Moore, 1996).

La clasificación de la Cirrosis ha sido un tema tratado desde distintos puntos de vista. Aunque el tamaño de los nódulos puede sugerir la Etiología, no deben utilizarse los términos micronodular y macronodular como clasificación básica ya que muchas formas de Cirrosis, sobre la alcohólica, son inicialmente micronodulares, pero los nódulos tienden a aumentar de tamaño con el tiempo, lo que se contrarresta por las limitaciones impuestas por las cicatrices fibrosas (Colectivo de autores, 1996).

Existe además otra clasificación fundamentada en la evaluación ultrasonográfica y determinación de alfafeto- proteína dándose las diferentes categorías de Cirrosis, de acuerdo con la intensidad y magnitud de los parámetros previamente establecidos en $\mathrm{A}$, B y C; clasificación de Child-Pugh. La misma presenta limitaciones ya que guarda mucho peso la etiología alcohólica. (Melo et al., 1998; Almeida et al., 1998).

Las principales consecuencias clínicas han sido: Ascitis, formación de cortocircuitos venosos portosistémicos, Esplenomegalia congestiva y Encefalopatía hepática. Otros elementos claves en cuanto al contexto clínico, corresponde a los shunt anatómico- 
intrapulmonares que condicionan a alteraciones de la vasculatura pulmonar generando hipoxemia arterial (García, 1997).

De igual manera, en la Cirrosis Hepática, la biopsia por vía percutánea constituyó en la última década del siglo XX, el método diagnóstico de elección. De manera general, constituía un procedimiento sencillo, sin riesgos, barato y fácilmente aceptable por el paciente. Como complementos, se establecieron marcadores séricos de fibrogénesis (PIII$\mathrm{P})$, pues dichos niveles se encontraban significativamente aumentados únicamente cuando la enfermedad hepática llegaba a ser clínicamente evidente (De la Maza, et al., 1997). Se invocaba además la evaluación radiológica de la circulación esplácnica portal con el uso de prostaglandinas (Medman, Azzo, et al., 1997).

A partir de la notable repercusión a nivel provincial de la Cirrosis Hepática, se realiza el presente trabajo con el objetivo de describir las principales causas de muerte y hallazgos necrópsicos en fallecidos por Cirrosis Hepática en el Hospital Provincial Clínico Quirúrgico Docente "Saturnino Lora", en Santiago de Cuba, República de Cuba, durante el decenio de 1991-2000.

\section{Marco Teórico}

En su clásica monografía, Laënnec describía el cuadro patológico de la Cirrosis y algunas de sus características clínicas, indicando que el proceso debería denominarse "Cirrosis" (Laënnec, 1826). El término de Cirrosis Hepática proviene de la palabra griega "Xippos", que significa amarillo naranja, fue, por tanto, el color leonado del hígado más que su consistencia el que dio el nombre a la enfermedad. Dicho autor adoptó la denominación de "Cirrosis", impresionado por el color del hígado. Posteriormente se propuso que "cirrosis" sugería con más profundidad «escirro»; que significa nodular y duro. Con el paso del tiempo la "Cirrosis" se ha identificado también con la cicatrización más que coloración del hígado (Bockus, 1980).

Se ha escrito mucho más acerca de la definición de cirrosis. En gran aparte de la confusión y la controversia que ha existido acerca de su definición, persistía la tendencia casi compulsiva al incluir diversos aspectos de su patogenia, que es aún menos conocida que la propia definición. Conn M.D, Harold G. (1980). La Cirrosis puede considerarse desde el punto de vista de los datos anatomapatológicos existentes acerca del hígado caracterizado por una destrucción difusa y una regeneración de las células del parénquima hepático y en las que existe también un aumento difuso del tejido conectivo que da lugar a una desorganización de la arquitectura lobulillar (Schiff, 1980).

Al efecto del presente trabajo, se asume que la Cirrosis Hepática, es la alteración anatomopatológica que se origina por la alteración difusa del parénquima hepático. Se caracteriza por regeneración de las células en forma de nódulos y aumento difuso del tejido conectivo, lo que se expresa por bandas de fibrosis (Castellanos, 2012). Esta enfermedad llegó a constituir una de las diez primeras causas de muerte en Cuba, en un rango de 8 a 10 por cada 100000 habitantes (Castellanos, 2012). 
Aunque en gran medida se debía al consumo de alcohol, otros factores importantes que contribuían a su desarrollo eran la hepatitis crónica, la enfermedad biliar y la sobrecarga de hierro. Este estado terminal de las hepatopatías crónicas aún manifiesta características específicas: Primeramente, los tabiques fibrosos en forma de bandas delicadas que unen las distintas estructuras portales o centrolobulillares, o en forma de amplias cicatrices que sustituyen a varios lobulillos adyacentes. En segundo lugar, nódulos parenquimatosos, causados por la regeneración de los hepatocitos que quedaron aislados, de tamaño variable, desde pequeños ( $<3 \mathrm{~mm}$ de diámetro) o micronódulos.

Debe insistirse en varios aspectos: Primero, la lesión parenquimatosa y la fibrosis consiguiente son difusas y se extienden por todo el hígado; las lesiones focales con cicatrización no constituyen Cirrosis. Para poder establecer el diagnostico, son imprescindibles los nódulos, reflejo del equilibrio entre la actividad regeneradora y la cicatrización constrictiva. La fibrosis una vez establecida suele ser irreversible, aunque en casos raros de esquistosomiasis y hemocromatosis se ha observado regresión. Y finalmente existe una reorganización de la arquitectura vascular, secundaria a la lesión, parenquimatosa y a la cicatrización con formación de comunicaciones animales entre los vasos que llevan la sangre al órgano y los canales venosos de salida (Robbins y Cotran, 2000).

\section{Metodología}

La investigación realizada fue de tipo descriptiva, transversal y retrospectiva con el objetivo de describir las principales causas de muerte y hallazgos necrópsicos en fallecidos por Cirrosis Hepática en el Hospital Provincial Clínico Quirúrgico Docente "Saturnino Lora" durante el decenio de 1991-2000. La muestra intencional, estuvo constituida por 137 fallecidos necropsiados cuya causa básica de muerte resultó ser la Cirrosis Hepática en el período señalado anteriormente según protocolo de necropsia. En este sentido, se procedió a evaluar diferentes variables de interés de la siguiente forma:

$1^{\circ}$ La determinación de frecuencia de necropsia. Se obtuvo mediante la aplicación de la siguiente ecuación:

$\%$ Frecuencia de Necropsia $=($ Total de necropsias a fallecidos $) /($ Total de necropsia en el hospital por año) $* 100$

$2^{\circ}$ La determinación de los años de vida potencialmente perdidos. Para ello se procedió a determinar el punto medio de cada clase, posteriormente se obtuvo la diferencia entre el punto medio de cada clase y el promedio de vida para la población cubana, resultado que se multiplicó por el número de fallecidos de cada grupo etáreo lo que resultó en $\Sigma$ AVPP.

$3^{\circ}$ La determinación de la tasa de años de vida potencialmente perdidos (AVPP). Se procedió a la aplicación de la siguiente ecuación:

Tasa AVPP $=(\Sigma$ AVPP $) /($ Total de población en estudio $)$ 
$4^{\circ}$ La determinación de la edad como variable cuantitativa. Se consideró al número de años cumplidos antes del fallecimiento. Información que se obtuvo de la historia clínica y el protocolo de necropsia y se dividieron en diferentes grupos etáreos de 15-34, de 35-54, de 55-74 y de 75-94 años.

$5^{\circ}$ La determinación del sexo, como variable cualitativa nominal, la cual se evaluó atendiendo a sus dos categorías, según género, masculino y femenino. Información extraída de la Historia Clínica y protocolo de necropsia.

Para conocer la relación existente entre la edad y la estadía hospitalaria, se consideró esta última como el tiempo que permaneció el enfermo recibiendo atención médica desde su admisión en el hospital hasta la fecha de su fallecimiento, variable que se interrelacionaron entre sí. Se dividió en los rangos: 1-7 días, 8-21 días, de 22-60 días y más de 60 días, información obtenida de Historia Clínica y protocolo de necropsia.

Para definir las categorías ocupacionales, la misma se evaluó como variable cualitativa nominal con cuatro categorías básicas:

1. Jubilados: Sujetos que permanecieron vinculados a determinada actividad laboral socialmente útil y que por su edad establecida o enfermedad intercurrente no continuaron la misma.

2. Amas de casa: Mujeres que se dedicaron a las labores del hogar.

3. Trabajador: Se agruparon a los sujetos que en el momento de fallecer brindaban actividad laboral socialmente útil, independientemente de su profesión y con edad superior.

4. Desocupado: Individuos sin vínculo laboral conocido.

La anterior información se obtuvo de la historia clínica. Para la determinación de la causa directa de muerte se consideró como variable cualitativa todo proceso mórbido que provocó directamente el fallecimiento del enfermo. Dato obtenido del protocolo de necropsia. Para conocer los principales hallazgos necrópsicos, variable cualitativa, se consideraron todas aquellas categorías subyacentes que se presentaban en un fallecido por Cirrosis Hepática y que fueran independiente de la causa directa de la muerte, pero podían comprometer la vida del enfermo. Esta información se obtuvo del protocolo de necropsia.

La recolección del dato primario se llevó a cabo a través de una planilla de vaciamiento de datos confeccionada al efecto. Se utilizaron fuentes de información secundarias como la historia clínica y protocolo de necropsia disponibles en el departamento de archivo y anatomía patológica. El procesamiento de los datos específicos se realizó a través de cuadros de contingencia estadística para darle salida a la información, se interrelacionaron variables. Los resultados se expresaron en frecuencias absolutas y relativas para variables cualitativas. Se utilizó el Chi cuadrado con un rango de significancia de $\mathrm{P}<0.05$. Además, se empleó el promedio para series agrupadas. Finalmente, se realizó el análisis de los resultados obtenidos y se compararon los mismos con otros estudios nacionales e internacionales lo que permitió emitir conclusiones al respecto. Los resultaron fueron expresados en cuadros estadísticos. 


\section{ORANGE JOURNAL}

\section{Resultados}

$\mathrm{Al}$ evaluar las principales causas directas de muerte en una determinada enfermedad es fundamental la identificación de las mismas por medio de la necropsia. En la Tabla 1 se muestra la frecuencia de necropsia en fallecidos por Cirrosis Hepática, en los primeros cuatro años del período evaluado, la cual tuvo un comportamiento variable, sin embargo, a partir de 1995 se comprobó un incremento significativo de forma relativa hasta el año 2000 , definiéndose con frecuencia de $0,85 \%$ para el decenio. Se consideró, que en ese período, se realizaron 16061 necropsias a diferentes categorías nosológicas, previamente definidas según la clasificación internacional de enfermedades.

Tabla 1.

Frecuencia de necropsias en fallecidos por Cirrosis Hepáticas. Hospital Provincial Saturnino Lora. 1991-2000.

\begin{tabular}{|c|c|c|c|}
\hline Año & Total de Necropsias & $\begin{array}{r}\text { Total de Necropsias } \\
\text { Fall X CH }\end{array}$ & $\%$ \\
\hline $\mathbf{1 9 9 1}$ & 1554 & 8 & 0.51 \\
\hline $\mathbf{1 9 9 2}$ & 1524 & 9 & 0.59 \\
\hline $\mathbf{1 9 9 3}$ & 1814 & 7 & 0.38 \\
\hline $\mathbf{1 9 9 4}$ & 1972 & 8 & 0.40 \\
\hline $\mathbf{1 9 9 5}$ & 1906 & 20 & 1.04 \\
\hline $\mathbf{1 9 9 6}$ & 1794 & 19 & 1.05 \\
\hline $\mathbf{1 9 9 7}$ & 1629 & 17 & 1.04 \\
\hline $\mathbf{1 9 9 8}$ & 1020 & 14 & 1.37 \\
\hline $\mathbf{1 9 9 9}$ & 1294 & 17 & 1.31 \\
\hline $\mathbf{2 0 0 0}$ & 1554 & 18 & 1.15 \\
\hline Total & $\mathbf{1 6 0 6 1}$ & $\mathbf{1 3 7}$ & $\mathbf{0 . 8 5}$ \\
\hline
\end{tabular}

Fuente: Departamento de Estadísticas.

El nivel y calidad de vida de un determinado grupo poblacional se determinó a través de diferentes parámetros, como el promedio de vida de un individuo al nacer. Esto obligó a la determinación de los años de vida potencialmente perdidos (AVPP), teniendo que en cuenta que la Cirrosis Hepática es una enfermedad de compartimiento crónico y que en determinado momento de su evaluación puede provocar la muerte. En Cuba, la esperanza de vida al nacer ha sido de 75 años como promedio. En el período estudiado, se perdieron 1220 años de vida potencial por esta causa con una tasa de 8,9. Estos resultados se muestran en la Tabla 2.

Tabla 2.

Años de vida potencialmente perdidos en fallecidos por Cirrosis Hepática. Hospital Saturnino Lora. 1991-2000

\begin{tabular}{|c|c|}
\hline AVPP & 1220 \\
Tasa & 8,9 \\
Edad promedio & 75 años \\
\hline
\end{tabular}

Fuente: Elaborado por los autores. 
En la Tabla 3, se muestra la distribución de los fallecidos según grupos de edades y sexo, el paciente que menos edad tuvo fue de 15 años y el mayor 94, la edad promedio de la muestra estudiada fue de 75 años. Los grupos etáreos de mayor mortalidad fueron a partir de los 35 años siendo, el más representativo el de 55-74 años representado por el 35,75 $\%$ del total, la mortalidad fue menos manifiesta en individuos jóvenes (15-34 años) la que representó solo el $11.00 \%$, como se puede apreciar se encontró un significativo predominio del sexo masculino $(54,78 \%)$ sobre el femenino $(42,22 \%)$.

Tabla 3.

Fallecidos por Cirrosis Hepática según grupos de edades y sexo. Hospital Saturnino Lora. 1991-2000

\begin{tabular}{|c|c|c|c|c|c|c|}
\hline \multirow{2}{*}{ Grupos de } & \multicolumn{2}{|c}{ Masculino } & \multicolumn{2}{c|}{ Femenino } & \multicolumn{2}{c|}{ Total } \\
\cline { 2 - 8 } & No. & $\%$ & No. & $\%$ & No. & $\%$ \\
\hline Edades & & & & & & \\
\hline $\mathbf{1 5 - 3 4}$ & 8 & 5.90 & 7 & 5.10 & 15 & 11.00 \\
\hline $\mathbf{3 5 - 5 4}$ & 20 & 14.59 & 17 & 12.40 & 37 & 26.99 \\
\hline $\mathbf{5 5 - 7 4}$ & 29 & 21.16 & 20 & 14.59 & 49 & 35.75 \\
\hline $\mathbf{7 5 - 9 4}$ & 18 & 13.13 & 18 & 13.13 & 36 & 26.26 \\
\hline Total: & $\mathbf{7 5}$ & $\mathbf{5 4 . 7 8}$ & $\mathbf{6 2}$ & $\mathbf{4 5 . 2 2}$ & $\mathbf{1 3 7}$ & $\mathbf{1 0 0 . 0 0}$ \\
\hline
\end{tabular}

$\mathbf{P}<\mathbf{0 . 0 5} \mathrm{X}=75$ años

Fuente: Historia Clinica y Protocolos de Necropsias

La distribución de fallecidos por Cirrosis Hepática según categoría ocupacional se muestra en la Tabla 4. La mortalidad estuvo concentrada en individuos con categoría ocupacional de trabajadores con el $34.30 \%$ seguido de las amas de casa (24.85\%) y los desocupados con $22.62 \%$, los menos afectados resultaron ser los estudiantes con solo el $2.91 \%$ del total.

Tabla 4.

Fallecidos por Cirrosis Hepática según categoría ocupacional. Hospital Saturnino Lora. 1991-2000.

\begin{tabular}{|c|c|c|}
\hline Categoría ocupacional & No. & $\%$ \\
\hline Trabajadores & 47 & 34.30 \\
\hline Amas de casa & 34 & 24.85 \\
\hline Desocupados & 31 & 22.62 \\
\hline Jubilados & 21 & 15.32 \\
\hline Estudiantes & 4 & 2.91 \\
\hline Total & $\mathbf{1 3 7}$ & $\mathbf{1 0 0 . 0 0}$ \\
\hline
\end{tabular}

Fuente: Historias Clínicas y Protocolos de Necropsias.

En relación a la estadía hospitalaria de los fallecidos (Tabla 5) el 54.76\%, fueron a óbito durante los primeros siete días de evolución en el hospital, 44 enfermos fallecieron entre 8 y 14 días para el $32.11 \%$ y solo el $13.13 \%$ lograron sobrepasar los 15 días de estadía. En la literatura consultada se reporta que la evolución y pronóstico de la Cirrosis Hepática es variable y se citan supervivencias superiores a los 5 años y generalmente la muerte 
sobreviene bajo situaciones interrecurrentes en dependencia del deterioro de la función y metabolismo hepático del afectado. En el caso particular de Cuba, la posibilidad de desarrollar una descompensación de la enfermedad ha sido de un 40 y $60 \%$ a los 5 y 10 años respectivamente de habérseles diagnosticado la enfermedad (Castellanos, 2012).

Tabla 5.

Fallecidos por Cirrosis Hepática según estadía hospitalaria. Hospital Saturnino Lora. 1991-2000.

\begin{tabular}{|c|c|c|}
\hline Estadía hospitalaria & No. & $\%$ \\
\hline $\mathbf{1}-\mathbf{7}$ días & 75 & 54.76 \\
\hline $\mathbf{8}-\mathbf{1 4}$ días & 44 & 32.11 \\
\hline $\mathbf{1 5}-\mathbf{2 1}$ días & 18 & 13.13 \\
\hline Total & $\mathbf{1 3 7}$ & $\mathbf{1 0 0 . 0 0}$ \\
\hline
\end{tabular}

Fuente: Protocolo de Necropsias

Existen múltiples y variados procesos capaces de provocar la muerte de forma directa en individuos afectados por Cirrosis Hepática. En la Tabla 6 se citan las causas directas de muerte más importantes en la muestra evaluada. La insuficiencia hepática aguda resultó ser la más representativa con el $42.33 \%$, le siguió la Bronconeumonía Bacteriana (19.00\%) y Anemia Aguda (11.70 \%) respectivamente. Menos significativos lo constituyeron el Coma hepático $(5.83 \%)$, Sepsis generalizada (3.64 \%) y el Desequilibrio hidroelectrolítico con 2.98\%. Llamó la atención que solo el Síndrome Hepatorrenal y el Edema Agudo del pulmón estuvieron representados por un solo afectado en cuanto a causa de muerte se refiere, constituyendo el $0.72 \%$ del total.

Tabla 6.

Fallecidos por Cirrosis Hepática según causa directa de muerte. Hospital Saturnino Lora. 1991-200.

\begin{tabular}{|l|c|c|}
\hline \multicolumn{1}{|c|}{ Causa directa de muerte } & No. & $\%$ \\
\hline Insuficiencia hepática aguda & 58 & 42.33 \\
\hline Bronconeumonía bacteriana & 26 & 19.00 \\
\hline Anemia aguda & 16 & 11.70 \\
\hline No precisada & 13 & 9.50 \\
\hline Coma hepática & 8 & 5.83 \\
\hline Sepsis generalizada & 5 & 3.64 \\
\hline Desequilibrio hidroelectrolítico & 4 & 2.92 \\
\hline Tromboembolismo pulmonar & 3 & 2.18 \\
\hline Meningoencefalitis bacteriana & 2 & 1.46 \\
\hline Síndrome hepatorrenal & 1 & 0.72 \\
\hline Edema agudo del pulmón & 1 & 0.72 \\
\hline \multicolumn{1}{|c}{ Total } & $\mathbf{1 3 7}$ & $\mathbf{1 0 0 . 0 0}$ \\
\hline
\end{tabular}

Fuente: Protocolo de Necropsias 
En la Tabla 7 se muestran los hallazgos necrópsicos más importantes encontrados en la muestra evaluada, llamando la atención que la Ascitis representó el $29.19 \%$ seguida por la Esplenomegalia (18.97 \%), Ateroesclerosis grado III de la aorta y sus ramas (18.24\%) y las varices esofágicas con el $13.86 \%$. Menos significativos resultaron la Esofagitis aguda $(5.83 \%)$, Congestión pulmonar $(5.10 \%)$, Esteatosis hepática (4.37\%) y la Gastritis crónica con $4.10 \%$ entre otras.

Tabla 7.

Hallazgos necrópsicos más frecuentes en fallecidos por Cirrosis Hepática. Hospital Saturnino Lora. 1991-2000.

\begin{tabular}{|l|c|c|}
\hline Hallazgos necrópsicos & No. & $\%$ \\
\hline Ascitis & 40 & 29.19 \\
\hline Esplenomegalia & 26 & 18.97 \\
\hline Ateroesclerosis & 25 & 18.24 \\
\hline Varices esofágicas & 19 & 13.86 \\
\hline Atelectasia pulmón postero basal & 18 & 13.13 \\
\hline Ateroesclerosis coronaria grado III & 16 & 11.67 \\
\hline Nefroesclerosis & 10 & 7.29 \\
\hline Impregnación ictérica & 9 & 6.56 \\
\hline Esofagitis aguda & 8 & 5.83 \\
\hline Hemorragia gastrointestinal & 7 & 5.10 \\
\hline Congestión pulmonar & 7 & 5.10 \\
\hline Esteatosis hepática & 6 & 4.37 \\
\hline Gastritis crónica & 6 & 4.37 \\
\hline Hipertensión arterial & 5 & 3.64 \\
\hline Hepatitis crónica activa & 3 & 2.18 \\
\hline
\end{tabular}

Fuente: Protocolo de Necropsias

Los diferentes tipos de Cirrosis encontrados en la actual investigación se muestran en la Tabla 8, la Cirrosis Portal resultó ser la más frecuente con el $53.30 \%$, seguida por la postnecrótica representada por el $24.10 \%$, las menos frecuentes lo constituyeron la Biliar Primaria con solo 5 casos (3.64 \%) y la secundaria a congestión pasiva crónica del hígado derivada de in suficiencia cardíaca con igual porcentaje de presentación.

\section{Tabla 8.}

Fallecidos por Cirrosis Hepática según clasificación citológica. Hospital Saturnino Lora. 1991-2000.

\begin{tabular}{|l|l|l|}
\hline Tipos de Cirrosis & No. & $\%$ \\
\hline Cirrosis Portal & 73 & 53.30 \\
\hline Cirrosis post-necrótica & 33 & 24.10 \\
\hline Otros tipos de cirrosis no clasificadas & 12 & 8.76 \\
\hline Cirrosis post hepática & 9 & 6.56 \\
\hline Cirrosis biliar primaria & 5 & 3.64 \\
\hline Cirrosis por congestión & 5 & 3.64 \\
\hline Total & $\mathbf{1 3 7}$ & $\mathbf{1 0 0 . 0 0}$ \\
\hline
\end{tabular}

Fuente: Protocolo de Necropsias 


\section{Discusión}

El resultado del estudio de la frecuencia de necropsias en fallecidos por Cirrosis Hepática y los años de vida potencialmente perdidos en fallecidos por esta enfermedad, presenta similitud con estudios de Navarro Robles y colaboradores al evaluar la mortalidad por Cirrosis en individuos alcohólicos en México en el período 1991-1993 (Navarro et al., 1997). Dichos resultados son significativos para el establecimiento de comparaciones entre las cifras obtenidas y lo que ocurre en la actualidad, más cuando se tiene en cuenta que la población mayor de 65 años había superado el 13\% de crecimiento (Caled et al. 2000).

Por otra parte, Willianet et al. (1997), en un estudio en Jamaica, encontraron predominio del sexo femenino sobre el masculino y la mortalidad se comportó en un rango de edad comprendido desde uno hasta 72 años con un promedio de 48 años en la muestra evaluada y atribuyó sus resultados a que las mujeres tuvieron mayor relación con el consumo de alcohol y antecedentes de Hepatitis sérica. Ribeiroet et al. (1997) al analizar el comportamiento de cirróticos con disturbios de la coagulación a los que se les administró Antitrombina III, encontró que los fallecidos tuvieron edad promedio de 44 años y que su base etiológica resultó ser el alcoholismo.

Se considera que en el presente trabajo el predominio del sexo masculino obedezca a que estudios epidemiológicos realizados en nuestro país arrojan un evidente y representativo consumo de alcohol y mayor incidencia de hepatopatías crónicas que a la postre evolucionan a la Cirrosis Hepática. De igual manera, que la elevada mortalidad obedezca a que las personas, disponen del consumo de bebidas alcohólicas, si se tiene en cuenta la estrecha relación entre alcoholismo y cirrosis, resultado controvertido con relación a los agrupados en la categoría de estudiantes. Aunque no está de todo demostrado sí existen estudios que demuestran relación causa efecto.

Aunque la investigación no tuvo el propósito de identificar la relación alcohol- cirrosis, sí es importante destacar que, fundamentado en análisis hipotéticos, el alcohol es directamente hepatotóxico y que se incrementa su efecto nocivo en condiciones de malnutrición, condicionando prominentes cambios grasos, depósito intrasinusoidal de colágeno y ocasionalmente tumefacción celular Oliveira et al. (1997), afirmaron que el Síndrome Hepatorrenal constituyó la causa de muerte en pacientes cirróticos con precaria reserva funcional del parénquima hepático a los que se le realizó trasplante de hígado y fundamentó además que este fenómeno guardó relación con la interrelación HígadoRiñón ya que en los evaluados no se constató de forma significativa la presencia de hipotensión arterial e hipovolemia. Soto Henriquez (1997), al evaluar las causas directas de muerte en pacientes cirróticos planteó que el Síndrome Hepatorrenal es una de las complicaciones de la Cirrosis Hepática avanzada, es una insuficiencia renal aguda funcional habitualmente irreversible y de curso fatal, la cual se debería a una vasoconstricción cortical renal con aumento de sustancia vasoconstrictoras endógenas (angiotensina, epinifrina y endotelinas) y disminución de las vasodilatadoras (prostaglandinas y Kalicreinas). 
Las infecciones también constituyeron causas de muerte por Cirrosis. En la serie realizada se encuentra que la Bronconeumonía Bacteriana ocupó un lugar representativo y se opina que esto obedezca a episodios de bacteriemia prolongado fundamentalmente por gérmenes gramnegativos. En este caso, se tiene en cuenta que en la Cirrosis Hepática existe sustitución de las células del parénquima por tejido fibrótico y/o nódulos de regeneración, comprometiendo la función de síntesis de proteínas como la albúmina.

Además, la Bronconeumonía era contraída por enfermos crónicos y se comportó terminal al ser hospitalizado el paciente y las bacterias intrahospitalarias solían ser resistentes a los antibióticos. Freitas (1997), al evaluar 32 enfermos con Cirrosis encontró que los mismos desarrollaron Peritonitis Bacteriana espontánea, resultado que se relaciona con lo reportado en este estudio. Similares hallazgos han sido afirmados por otros autores (Martínez et al. 1997).

La Anemia Aguda como causa de muerte estuvo relacionada con los fenómenos derivados de la hipertensión portal, tales como ruptura de várices esofágicas o sangramiento hemorroidal u otros sitios como peritoneo, intestino delgado y colon. También, Campillo et al. (1997), al evaluar las características epidemiológicas de la Cirrosis Hepática en el hospital civil de Guadalajara encontró que la Ascitis junto a las hemorragias digestivas, constituyeron hallazgos notables en la muestra evaluada, resultado que coincide con la presente investigación.

Se opina que el elevado porcentaje de frecuencia de la Ascitis obedezca fundamentalmente a la hipertensión portal, así como de un incremento de la reabsorción de sodio por el riñón, lo cual es debido a una interferencia con el flujo portal en el hígado cirrótico, así como un incremento del volumen plasmático visceral o un desbalance en las fuerzas de Starling que gobiernan el normal intercambio de fluido entre el compartimiento vascular y tisular. Además, puede ser resultado de infecciones o tumoraciones malignas, Gitinet et al. (1997), Levy \& Wexler (1984). Michaely et al. (1997), encontraron que la Esplenomegalia se presentó en el 33\% de los fallecidos por cirrosis.

En cuanto a la Ateroesclerosis grado III, la cual constituye un endurecimiento de las arterias, en la actual investigación resultó ser un hallazgo necrópsico notable y está relacionado con la edad a que se inicia en la infancia y empeora por decenios en cualquiera de sus variantes. Resultado que coincide con lo reportado por otros autores (Kannel \& Wilson, 1995; Strong, 1991).

De igual manera, en el presente trabajo, se considera que la elevada frecuencia de la Cirrosis Portal esté relacionada en que la misma en su gran mayoría obedece al consumo excesivo y prolongado de bebidas alcohólicas situación ya abordada anteriormente.

Por su parte Garzón et al. (1998) demostraron la presencia de Cirrosis Hepática en individuos con exposición prolongadas a drogas hepatotóxicas, la baja frecuencia de Cirrosis biliar primaria pudiera estar en relación con su baja incidencia de presentación en la población, casi exclusiva en las mujeres y estrechamente vinculadas a procesos inmunológicos. Rodríguez et al. (1997) reportó un caso de Cirrosis biliar primaria asociada a mieloma múltiple e hipotiroidismo. Estos aspectos pueden estar vinculados a 
las alteraciones del sistema inmune. Así, la Cirrosis por congestión podría obedecer a hipoxia hepática resultante de la disminución del flujo sanguíneo hepático siendo más afectado el área pericentral del lóbulo hepático.

\section{Conclusiones}

El estudio realizado acerca de las causas de muerte y hallazgos necrópsicos en fallecidos por Cirrosis Hepática en el Hospital Provincial Clínico Quirúrgico Docente "Saturnino Lora", en Santiago de Cuba, durante el decenio de 1991-2000, arrojó un incremento progresivo de la frecuencia de necropsia en fallecidos por Cirrosis Hepática, siendo más notable a partir de 1995, una tasa de años de vida potencialmente perdidos (AVPP) por encima de la media.

La mortalidad por Cirrosis Hepática fue más frecuente a partir de los 55 años, con predominio en el sexo masculino y en individuos con categoría ocupacional de trabajadores y amas de casa. En las muestras evaluadas, se comprobó que los primeros siete días de estadía hospitalaria constituyeron los de mayor mortalidad. En el universo estudiado la Insuficiencia Hepática, Bronconeumonía Bacteriana y la Anemia Aguda resultaron ser las principales causas de muerte. Se destacaron como hallazgos necrópsicos más representativos la Ascitis, Esplenomegalia, Ateroesclerosis de la Aorta y sus ramas grado III. La Cirrosis Portal y la post-necrótica constituyeron los tipos etiológicos más frecuentes encontrados en las muestras evaluadas.

\section{Referencias bibliográficas}

Almeida, J. M. R, Leite Mór, M. M. B., \& Parise, E. R. (1998). Determinación sérica de ácidos biliares en la detección de hepatopatía crónica y su estadiamiento funcional. Arq. Gastroenterol, 35(2), 81-8, http://bases.bireme.br/cgibin/wxislind.exe/iah/online/?IsisScript=iah/iah.xis\&src=google \&base=LILACS \&lang $=$ p\&nextAction $=\operatorname{lnk} \&$ exprSearch $=217975 \&$ indexSearch $=$ ID

Bockus, H.L. (1980). Gastroenterología. Tomo 3. Edición Revolucionaria. Editorial Científico Técnica. P. 395. Capítulo Cirrosis.

Caled, E., et al. (2000). Biología del envejecimiento. Parte III. Capítulo 5. P. 14. En: Cecil. Tratado de Medicina Interna. Vol I. Editorial Interamericana.

Campillo, O. et al. (1997). Características epidemiológicas de la cirrosis en el Hospital de Guadalajara. Salud P. Mexico, 39(3), 195-200.

Castellanos, M. I. (2012). Cirrosis hepática. En C. de autores. Diagnóstico y tratamiento en medicina interna. Capítulo 43, pp. 339-343. Cuba: Editorial Ciencias Médicas.

Colectivo de autores (1996). Tratado de Hepatología. Tomo II. 1996. Sexta Edición. Cuba: Editorial Ciencias Médicas,

Conn, M.D, \& Harold, G. (1980). Cirrosis. Enfermedades del hígado. L. Schiff. Capítulo 26, pp. 947. Editorial Científico Técnica. Tomo 2.

Creutzteldt, W, Beek, K. (1956). Cirrohosis of the liver: Ondeaerodogr, pathogenesis, results of tratment and periode of survival in an selected series of 560 patients. Germedmonth, 11, 259.

De la Maza, et al. (1997). Marcadores de fibrogenesis hepática en pacientes alcohólicos. Rev. Med. Chile, 125(1), 15-21. 
Freitas, I. (1997). Estudio comparativo entre 3 métodos de cultivo de líquido ascítico con neutrofilia. Geo Gastroenterolendosdig, 16(2), 37-40.

García, E., et al. (1997). Función pulmonar en cirrosis hepática. I. Pneunol, 23(5), 225-30.

Garzón, M. et al. (1998). Hepatotoxicidad en el Hospital Universitario de la Samaritana. Rev. Colm. Gastroenterol, 13(2), 73.-6.

Gitin, N. et al. (1997). The Ph of ascilis fluid in the diagnosis of spontaneous bacterial peritonitis in alcoholic cirrosis. Hepatology, 2, 408.

Kannel, W.B. \& Wilson, P.W. (1995). An uptade on coronary risk factor. Med. Clin. North am, 79, 951.

Laënnec, R.T.H (1826). Treaty of mediate auscitation. Paris: Chaude, pp. 196.

Levy, M. \& Wexler, M. (1984). Salt and wáter balance in liver disease. Hosp. Pract, 57.

Martínez, J., et al (1997). Peritonitis bacterianaespontánea. Rev. Bras. Clin, 24(1), 33-6.

Martini, G.A. \& Bodec. (1970). The epidemiology of cirrohosis the liver. In. Engels a. Lorsson T: Alcoholic cirrhosis anthertoxishepatopatías. Stockholm: Nordiska Bokhandelns Forlag. 1970, pp. 315.

Medman, A., et al (1997). Splanchnic portal mcirculation: enhancement of the radiologgic image intensity and of the visibility of collateral sirroches with the use of prostaglondins El. Arq. Gastroenterol, 34(2), 97-104.

Melo, W., Coello, E.S.M., \& Moreira, D.M. (1998). Correlación de aspectos ultrasonográficos y clasificación de Child en la cirrosis hepática. Radiol Bras, 31(2), 83-92.

Michael, G. et al. (1997). Cirrosis hepática. West Indian Med. J., 46(2), 60-2.

Navarro, J. et al. (1997). Correlación ecológica entre consumo de bebidas alcohólicas y mortalidad por Cirrosis hepática en México. Salud P. Mexicana, 39(3), 217-20.

Oliveira, A. et al. (1997). Síndrome hepatorrenal luego de trasplantes de hígado. Consideraciones en 3 pacientes. Arq. Gastroenterol, 34(4), 235-40.

Ribeiro, A.A. et al. (1997). Uso de concentrado de antitrombina III en cirróticos con disturbios de la coagulación. Rev. AS Med. Bras, 43(3), 189-94.

Robbins y Cotran. (2000). Patología estructural y funcional. $6^{\text {ta }}$ Edición. Madrid: Editorial Interamericana Saunders. Capítulo 19, pp. 889-890.

Rodríguez, G., et al. (1997). Reporte de un caso de mieloma múltiple e hipertiroidismo en cirrosis biliar primaria. Rev. Inv. Clin, 49(3), 2154-20.

Schiff, M.D. (1980). Enfermedades del hígado. Capítulo 26, pp. 947. Edición Revolucionaria. La Habana: Editorial Científico Técnica. Tomo 2.

Soto, J. (1997). Síndrome Hepatorrenal. Bol Hosp. San Juan de Dios, 44(5), 284-6.

Strong, S.P. (1991). The natural history of ateroesclerosis in chilhood. An N. Y. Acad, 423, 9.

Van Roey, G. \& Moore, K. (1996). The hepatorrenal síndrome. PediatrNephol, 10, 100-107,

Willian, N.A., et al. (1997). Cirrosis hepática en Jamaica. West Indians Med. J, 46(2), 60-2. 